\title{
25 Years of Cancer Chemotherapy Pathways: A Brief History with a Look to the Future
}

\author{
J. Russell Hoverman \\ Quality Programs, Texas Oncology, Dallas, Texas, USA \\ Email: jrussell.hoverman@usoncology.com
}

How to cite this paper: Hoverman, J.R. (2021) 25 Years of Cancer Chemotherapy Pathways: A Brief History with a Look to the Future. Health, 13, 205-216.

https://doi.org/10.4236/health.2021.133018

Received: February 25, 2021

Accepted: March 7, 2021

Published: March 10, 2021

Copyright $\odot 2021$ by author(s) and Scientific Research Publishing Inc. This work is licensed under the Creative Commons Attribution International License (CC BY 4.0).

http://creativecommons.org/licenses/by/4.0/

\begin{abstract}
For patients receiving chemotherapy, drugs represent the largest cost. Clinical chemotherapy Pathways have become a critical strategy to identify unnecessary drug costs and to implement mechanisms to deliver lower cost alternatives without sacrificing outcomes or quality of care. This paper describes the steps of development of a functioning pathways program beginning in an environment of full-risk capitation, including drugs. The next steps involved quantitating the potential impact of such a program and then collaborating with a payer to test the concept. When these studies showed promise, the practices adopting pathways used them as a backbone for drug management in the Oncology Care Model. These experiences very likely represent steps in a continuum towards placing more of the drug delivery costs at risk. The potential for again considering capitated payments is discussed.
\end{abstract}

\section{Keywords}

Cancer Chemotherapy, Clinical Pathways, Drug Costs

\section{Introduction}

Clinical cancer chemotherapy pathways (Pathways) are now a critical component of Value-Based Care (VBC) for commercial insurers and Medicare. They came on the scene with an article published in 2010 seemingly out of whole cloth without an origins story. In fact, the journey for pathways began 15 years earlier. This paper recounts the data and principles that led to the development of the pathways program, the challenges faced in dissemination of the product and the implications of that experience for the future.

\section{Beginnings: Empirical Support for the Pathways Concept 1995-2005}

Texas Oncology $(\mathrm{TxO})$ is a professional association owned and operated by its 
physicians. In 1996 it had over 150 physicians and was expanding into new markets. Some markets were dominated by independent practice groups or insurers who were capitating services. In that model, providers were paid a fixed fee per covered patient per month (PMPM-per member per month) for a defined set of services. For primary care or integrated practices, this could cover the entire cost of services, including hospitalization and drugs. This is full risk and these providers could in turn sub-contract with specialty providers to deliver a range of services outlined in a capitation model with a PMPM (per member per month) payment for those services.

For $\mathrm{TxO}$, there was a recognition that expanding the referral base would involve risk in capitation dominated markets. Accordingly, $\mathrm{TxO}$ contracted with both providers and insurers to accept full risk for Medical Oncology, Malignant Hematology and Radiation therapy for cancer care. This included all outpatient and inpatient professional charges and outpatient drugs, both cytotoxic and supportive. By July 1996, over 100,000 lives were covered. From July 1998 to January 2000, over 500,000 lives were covered each year.

At that time, the only data $\mathrm{TxO}$ had to forecast costs were claims and population cancer incidence. There was no electronic health record. There was no access to hospital data. However, this was an enormous push to understand our resource utilization.

The two most common cancers were breast cancer and lung cancer. For breast cancer the most common use of drugs was in adjuvant chemotherapy. In this setting, there were four options: CMF PO (PO-by mouth, IV-intravenous) (cyclophosphamide PO, methotrexate IV and 5-flourouracil IV, 28 day cycles $\times 6$ ), CMF all IV 21 day cycles x 6, AC (Adriamycin, cyclophosphamide) IV 21 day cycles $\times 4$ and FAC IV 21 day cycles $\times 6$. The respective total costs for each regimen were $\$ 2694, \$ 4140, \$ 5096$ and $\$ 7654$. The clinical equivalence was evaluated and published by TxO breast cancer specialists [1]. As Adriamycin (generic-doxorubicin) containing regimens comprised the majority of regimens given, $\mathrm{AC}$ was chosen as the preferred regimen. $\mathrm{AC}$ had the benefit of lower cost than FAC and shorter duration of therapy.

This preference for breast cancer adjuvant chemotherapy was socialized among all physicians but especially among those with a large capitated population. Figure 1 shows the results of this activity with a large drop in FAC use and a concomitant rise in the use of $\mathrm{AC}$. As shown, there was a remarkable increase in $\mathrm{AC}$ in the capitated physician cohort.

For lung cancer, the most common clinical condition was metastatic nonsmall cell lung cancer. Influenced by the studies of Brent James and David Eddy [2], which demonstrated the variation in costs and lack of association of those costs with outcomes, the population of lung cancer patients was studied to correlate costs with outcomes.

For costs, there was a more than a two-fold difference in costs comparing the top quartile with the bottom quartile Figure 2 . The primary difference was in pharmacy cost as shown in Figure 3. Figure 4 indicates that higher drug cost 


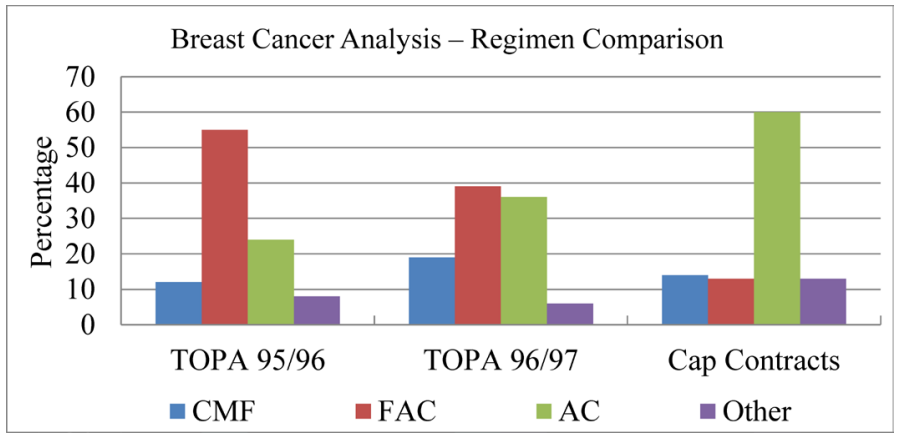

Figure 1. The distribution of regimen choice for breast cancer chemotherapy.

Figures for Lung Cancer A Retrospective Study

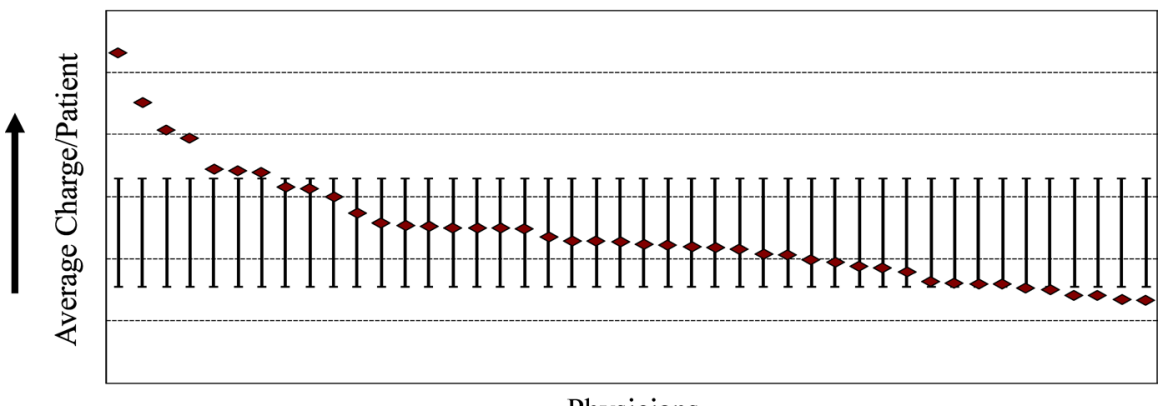

Physicians

Figure 2. The distribution of cost per case of lung cancer chemotherapy treatment by physician.

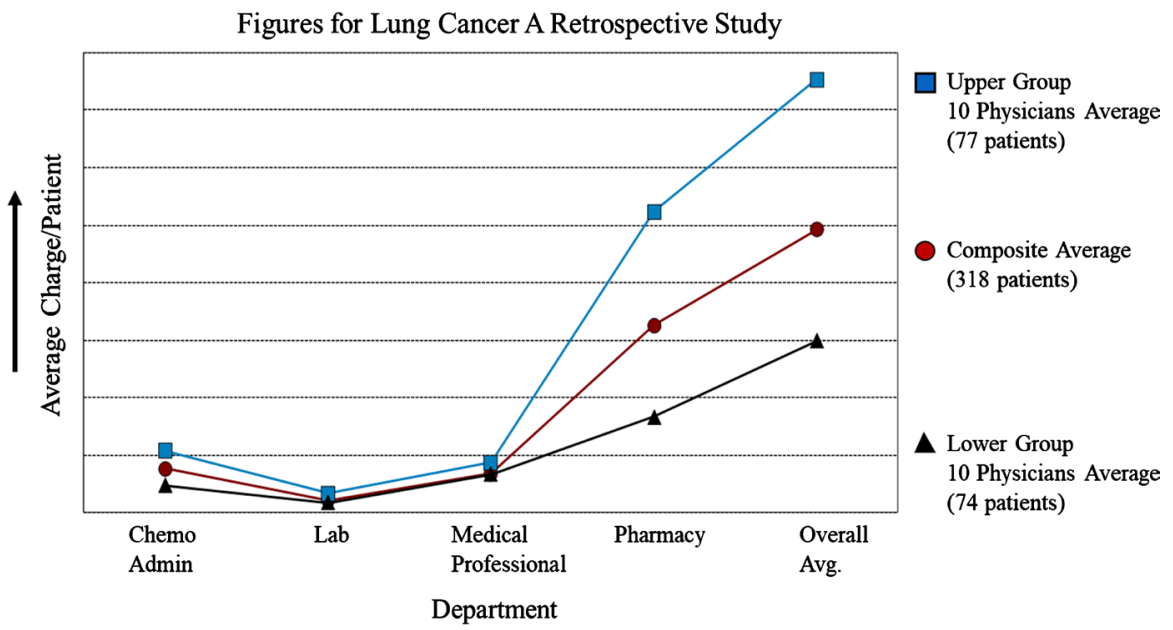

Figure 3. Costs by category for lung cancer chemotherapy sorted by the highest cost and the lowest cost.

was associated with later lines of therapy. A separate analysis demonstrated that the high pharmacy costs were associated with the relative high use of white and red cell growth factors as well. Figure 5 shows survival curves for both the top quartile for cost and the lowest quartile. There was no difference in survival. These findings allowed the practice to make recommendations regarding supportive drugs and lines of therapy to all physicians [3].

By the year 2000, the enthusiasm for capitation, and HMOs, waned and the 


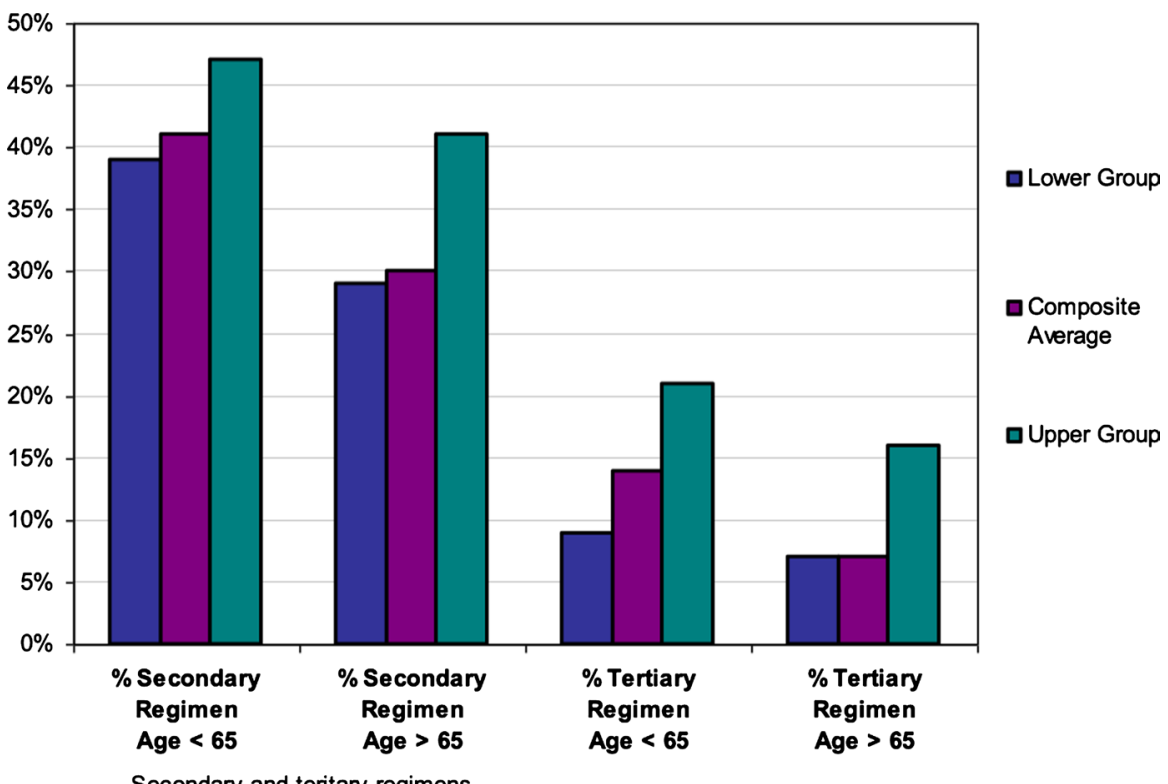

Figure 4. The percentage use of second and third-line chemotherapy for lung cancer sorted by highest cost group and lowest cost group.

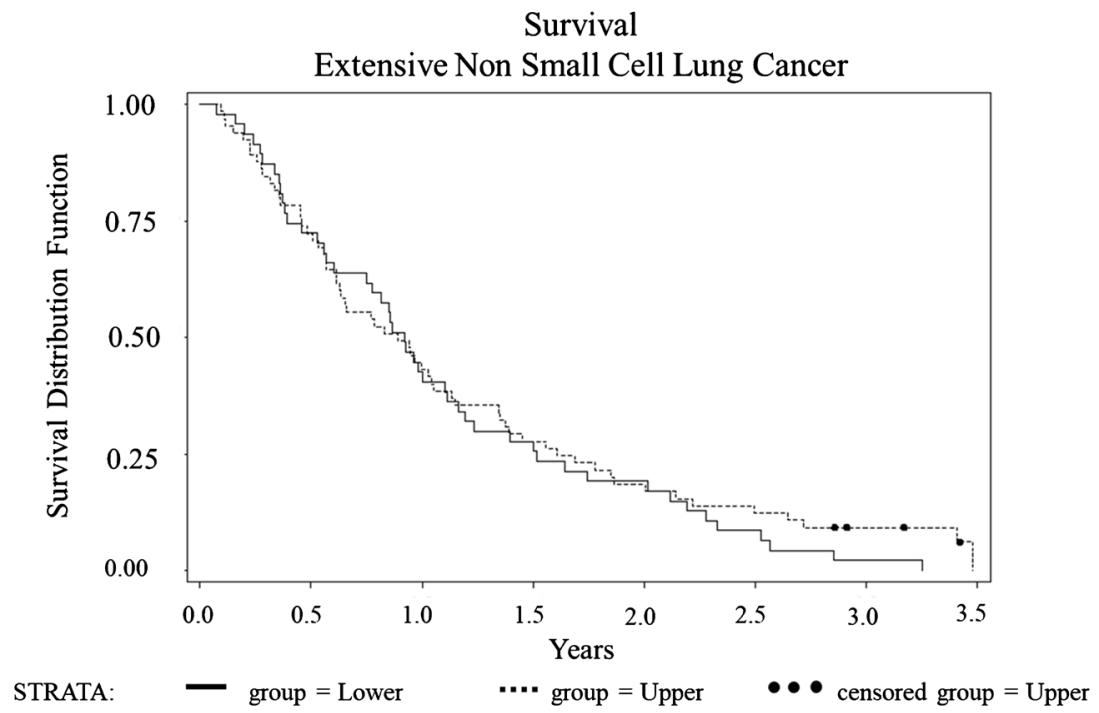

Figure 5. Comparison of survival curves with the highest cost cohort compared with the lowest cost cohort. $\mathrm{P}$ value for difference $=0.99$.

cancer specialty capitation arrangements rapidly unwound. However, there were important lessons learned: 1) Costs could be addressed without compromising outcomes. 2) Physician behavior could be impacted with cost and outcome data. 3) Managing a risk contract with last century technology was impossible. 4) Other important cost centers were not addressed. 5) The opportunities for study were abundant. There were other important attributes of this exercise: 1) This was a platform and not a program. 2) These were recommendations only. 3) There was no formal approval or exception process. 4) The TxO Quality Oversight Committee decided that drug choice could be made using a priority of 
outcomes first, toxicity second, cost to patient, payer and employer third and margin last.

Capitation was an enormous push for change. Once that push disappeared, change languished.

\section{Bringing Pathways to the Market Place: 2006-2015}

Yet, for the community cancer care provider, the oncology landscape began to change in profound ways. In 2003 with the passage of the Medicare Modernization Act, chemotherapy related drug net income earned by community oncologists was to drop $20 \%-30 \%$ for treating Medicare patients. This was accomplished by changing the Medicare reimbursement method from Average Wholesale Price to Average Sales Price. It was expected that commercial payers would adopt a similar approach.

The changes played out as expected, and in the 2011 National Practice Benchmark, a survey of mostly community oncology practices, the drug revenue as a percentage of total revenues dropped from $85 \%$ in 2005 to $65 \%$ in 2010 [4].

During this same period, community oncology practices were also disadvantaged compared to hospital systems, which receive significantly higher reimbursement for services and drugs [5]. These combined changes led to an exodus of oncologists from community-based, physician-owned practices to hospital-based, hospital-owned practices [6].

Some obvious responses could be made to address these economic challenges. For example, practices could diversify by adding additional services; they could improve clinical and business efficiencies; they could maximize employee contributions by ensuring responsibility at the skill level of training. In fact, all of these have been done, but the inescapable impression is that to survive a new basis for payment, the value of services delivered must be demonstrable.

Both TxO and The US Oncology Network (USON) were struggling to differentiate themselves based on quality and value. The situation changed dramatically with a presentation by Marcus Neubauer, MD, to a US Oncology Network national meeting in 2006 reporting the Kansas City Cancer Center's (KCCC) experience with a new pathways program. The physicians at KCCC realized that cancer drug costs were rising and relationships with payers were becoming more strained. They made the decision to make changes to enhance value. They developed clinical pathways for the common types of cancers. The idea was to evaluate all regimens and exclude those that were redundant or more expensive. They felt that by eliminating unnecessary variation, they could reduce errors, reduce cost, and improve efficiency.

The principles of the pathways program were simple; however, execution more difficult. Regimens for various lines of therapy were assessed, first by outcomes, second by toxicity. If equivalent on these counts, regimens were selected based on cost to patient and payer. Execution required general agreement among practice physicians to follow pathways, dissemination of the pathways to all 
personnel, and development of a real-time review process. The pathways were not designed to cover $100 \%$ of cases. Exceptions were allowed but a reason for the exception from the treating physician was required and this was reviewed by the KCCC peer-review committee and could either be approved or denied. The request could be denied if the explanation was unsupportable by a review of the clinical evidence.

The initial results of the Pathways program for KCCC are seen in Figure 6. With pathways, carboplatin/Taxol (paclitaxel) and clinical trials were $85 \%$ of all ordered regimens. There was negligible use of chemotherapy beyond second line treatment. Prior to pathways, in addition to paclitaxel-based regimens, there were significant proportions of use with docetaxel (Taxotere) and gemcitabine (Gemzar). Based on the experience of the KCCC, the clinical pathways program was supported and implemented by USON for use by the entire network nationwide. This started with a small team of physicians and pharmacists, the Pathways Task Force (PTF), charged with developing pathways for the major diseases, breast, colon, lung, and prostate cancers, and incorporating these into iKnowMed (iKM) electronic medical records (EMR) for decision support. A formal evidence base was created for each stage and each line of therapy for these diseases. Proposed pathways changes were then reviewed by each disease type research committee. Finally, comments were solicited from the entire network. The Pathways program now includes 19 tumor types imbedded into iKM.

Within the practice, iKM was augmented by Clear Value Plus (CVP) to facilitate data entry, collection and decision support. When appropriate data are input regarding disease (including biomarkers), stage and line of therapy, the pathways regimens automatically appear. If the physician wishes to use an off-pathway regimen, an exception must be requested, and a reason given through a defined field in CVP. The exception can be approved at the site level by a peer, and if not, the request is referred to a separate committee of physicians for review. This is an uncommon occurrence and the committee has not penalized physician

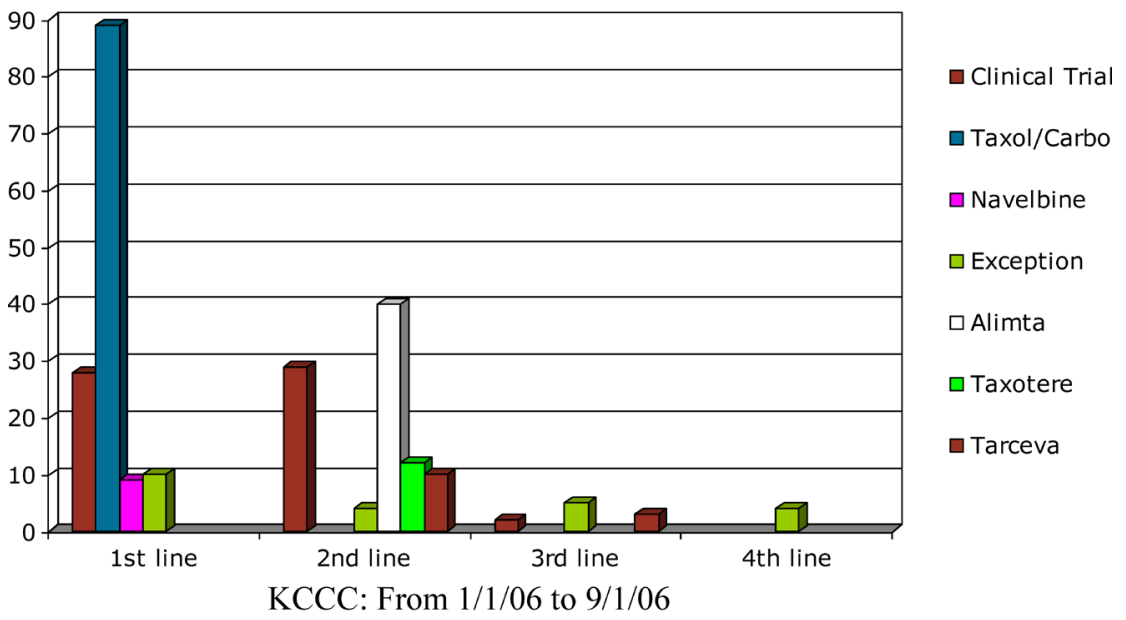

Figure 6. Regimen distribution for chemotherapy treatment for metastatic non-small-cell lung cancer following introduction of a Pathways program. 
ordering retrospectively, but this activity has led to a prospective list of drugs or regimens specific for disease stage and line of therapy that are not mixed if ordered. Overall pathways performance by site of service is sent out monthly to all centers. Individual physician data are available. Supportive education is in place and the practice allows a period of time for physicians to self-correct.

Undertaking the Pathways program was itself a risk. If successful, the income to the practice from drug margin would drop. In contrast to capitation, the practices themselves would have to push or pull the health system along to create a market for value-based care. It was left up to the Pathways team to push on two fronts: one: create the business case and two: expand the product. For the former, three studies were published. In the first, internal data from iKnowMed and billing records were evaluated to correlate pathways compliance with outpatient costs in non-small-cell lung cancer. This study showed that patients completely on-pathways had a reduction of approximately $35 \%$ in outpatient costs chiefly attributed to reductions in chemotherapy and growth factor use. There was no difference in survival between those on- and off-pathways [7].

In the second study, in collaboration with Milliman, the total costs for colon cancer were measured. All costs were tied to patterns of drug use consistent or inconsistent with pathways adherence. With internal iKM data, survival for both on- and off-pathways patients was measured. Once again, costs were lower in the pathways group for both adjuvant and metastatic diseases, with the reduction in costs encompassing both chemotherapy and hospital categories. There was no decrement in survival with adherence to pathways [8].

In a third study, in an extension of the Milliman collaboration, global costs for the major cancer cost categories were measured. The total average cost for chemotherapy patients was $\$ 111,000$ per year. $25 \%$ of this was attributable to drugs. Other major opportunities for cost reduction were management of chemotherapy toxicity to reduce hospitalizations and enhancement of end of life planning to reduce both drug and hospital costs [9].

These studies supported the potential of a pathways program to reduce costs while maintaining quality. They, particularly the second study with Milliman, suggested other areas of focus in an expanded program. The costs of care at the end of life are well-documented [10] [11]. At the same time, it became clear that active interventions, particularly involving improved communication, reduced expenditures, improved comfort, relieved stress for family members and did not shorten survival [12] [13] [14] [15] [16]. The USON team then developed a more comprehensive cancer management program that incorporated not only pathways, but an advance care planning component and a nurse call program (PSS-Patient Support Services) to improve end of life care and reduce hospitalizations from both chemotherapy complications and aggressive care. With the collaboration of Aetna, programs were piloted with $\mathrm{TxO}$ for both commercial [17] and Medicare Advantage (MA) populations [18]. The control group for the commercial pilot was TxO baseline costs. The control group for the 
MA pilot was concurrent non-TxO patients in Texas. Drug costs were flat over a three-year period for the commercial group, but hospital inpatient days were reduced by $44 \%$. In the MA pilot, drug costs were $21.4 \%$ and hospital costs $5.3 \%$ lower for TxO compared to controls. By the third year, hospice enrollment was $65 \%$.

USON was not alone in pushing towards value-based care. NCCN made evidence-based guidelines public for a wide range of cancers [19]. Barbara McAneny spearheaded the COME HOME project funded by the Center for Medicare and Medicaid Innovation (CMMI) [20]. Lee Newcomer led United Healthcare in an Episode of Care bundled payment program [21]. Others in other settings have found similar savings using clinical pathways [22] [23] [24].

In 2015, CMMI solicited applications for the Oncology Care Model (OCM). This was to be a program with shared savings if total cost of care was reduced. There were 6 overarching requirements: 24/7 physician coverage, routine use of the Institute of Medicine (IOM) Care plan, defined quality metrics, a system of patient navigation, a practice wide EMR and utilization of nationally recognized guidelines. This transformation was to be supported by payments for Monthly Enhanced Oncology Services (MEOS) on a monthly per enrolled patient basis.

\section{Taking the Next Steps: The Oncology Care Model and Beyond}

On July 1, 2016, 16 USON practices began enrolling patients into the OCM. Over 25,000 patients were enrolled in the Network each 6-month period. All practices were using the same EMR and the same pathways program (Value Pathways powered by NCCN). Originally a 5-year program, with the disruption of the COVID-19 epidemic it was extended to six. By the end of the $4^{\text {th }}$ year, 14 practices chose to stay in the program with the majority opting for 2-sided risk. Calculations using the network practices only indicate savings of over 100 million dollars for the first 6 periods or 3 years. The network has now seen over 100,000 patients (unpublished data).

The OCM represented an unprecedented opportunity for community oncology practices. Each practice had data for the total cost of care for each of their patients. This included non-oncology costs, hospital, ER, imaging, radiation, labs and hospice costs. Importantly, these data included reliable hospice admission dates and date and place of death.

Fortunately, data management tools have improved commensurate with the size of the data set so that analysis could be done down to the physician level. Physician drug use, hospitalization rate and hospice enrollment rate were all discoverable. The utilization of the IOM treatment plan can be tracked; pathways compliance can be measured and reported; novel patient navigation programs can be implemented, and the use of advance care planning enhanced.

Perhaps most importantly, being rewarded for certain kinds of behavior promotes a group ethic, in that poor performance hurts the performance of the 
group. It is a different way of measuring success and is closely tied to clinical and economic outcomes that benefit patients in measurable ways.

The practices in the US Oncology Network, through participation in the OCM and numerous other commercial and Medicare Advantage value-based arrangements, have been on the leading edge of transformation to a value-based healthcare delivery system. Yet there are numerous challenges to maintaining a leadership position in an unfavorable environment.

There are some obvious environmental challenges. The economic exigencies of the COVID-19 pandemic may have long lasting impacts on health care. There is a daunting national budget deficit as well as multiple states facing serious budget shortfalls. This will mean higher personal and corporate income taxes. It will also mean pressure on health care coverage for Medicare and Medicaid. The pandemic has only worsened the situation. In Texas over $25 \%$ of non-elderly adults do not have insurance with no prospects of expanding Medicaid [25]. With unemployment still high, more patients will be on Medicaid or uninsured. There will be enormous pressure on drug pricing, meaning that physician income on drug spend will drop along with the drug prices.

It is the price of products, not the costs of professional services that represent the overwhelming percentage of overall costs. Oncologists can be successful by managing the price of these products. These include drugs predominantly, but also laboratory studies, imaging, technology in all aspects of care, hospitalizations and emergency care.

A first step to anticipate these pressures is to make a very detailed evaluation of the economics of the OCM. If a practice has been successful, it has reduced drug use compared to the control population. This may mean it has sacrificed margin for performance. Looked at over a 5-year period, the net margin during the pre-pilot phase may have been preserved as the average price of drug has increased. The net income may also have been preserved by the MEOS payments. The impact of shared savings bonus payments is yet uncertain for most practices. It is critically important for each practice to know where the net income comes from, what the margins are on each product and whether those margins are sustainable. It may be that drug margins are higher, given the high cost of drugs, than they will ever be.

The plan for the OCM is to phase it out by the end of 2021. It will be replaced by the Oncology Care First model, which has not been finalized, but a capitated rate for Evaluation and Management codes (E\&M) including infusion charges has been proposed. Capitation is a risk, but also an opportunity. A capitation payment can include margin plus product cost. If the product cost is reduced and the payment stays the same, it enhances margin. However, if the cost of drugs rises, the net proceeds to the provider can drop precipitously. Given the cost of recently introduced new drugs, a capitation payment that includes drugs will be prohibitively risky [26]. It can also be a margin payment. For example, if the margin on drugs was capitated, the margin would stay fixed even though the 
total cost of drugs dropped. The proportion of price that is margin is much smaller than the cost of the drug so small investments in management would yield substantial returns.

For oncologists, there are other considerations that impact the value of care. The first is the inefficiencies of the drug delivery system. This can be addressed by evaluating the contribution of all vendors who touch a drug as it passes from manufacturer to patient. The second is the issue of co-payments for patients. If criteria were satisfied that the drug ordered was the most cost-effective for the patient's condition, reinforced by an effective Pathways program and a payment system that did not reward high drug prices, copays could be eliminated. This reduces the risk to the patient of financial toxicity, improves the likelihood of medication adherence and takes co-pay assistance programs out of the discussion of drug choice. Thirdly, all these activities may be for naught if the physician compensation structure does not mirror the payment structure in terms of case management, shared-responsibility, team-based care and performance-based payments.

Finally, pathways would provide a structure for even a worse-case scenario. The NCCN has a product called the Framework [27]. This is a pared down version of the standard guidelines designed for use in resource poor health care environments, particularly countries with limited economic resources. In the United States, we are faced with substantial inequities in income, education, opportunity and health care. Pathways would be a critical component of any Framework used to reduce drug costs to society.

\section{Conclusion}

Value-based chemotherapy pathways have become a critical element of value-based care. They have been validated in different environments and the concept adapted to other initiatives. There will be further changes to the cancer care delivery system with great pressure on drug prices. Pathways will continue to be the standard that will use evidence to protect patients.

\section{Conflicts of Interest}

The author declares no conflicts of interest regarding the publication of this paper.

\section{References}

[1] Blum, J.L., Jones, S., Fay, J.W., et al. (1997) Guidelines for Systemic Therapy of Early Stage Breast Cancer. Breast Cancer Research and Treatment, 43, 259-278. https://doi.org/10.1023/A:1005705300012

[2] James, B.C. and Eddy, D.M. (1994) CPI and Practice Guidelines. In: Horn, S.D. and Hopkins, D.S., Eds., Clinical Practice Improvement: A New Technology for Developing Cost-Effective Quality Health Care, Faulkner \& Gray, Washington DC, Chapter 9.

[3] Hoverman, J.R. and Robertson, S.M. (2004) Lung Cancer: A Cost and Outcome 
Study Based on Physician Practice Patterns. Disease Management 7, 112-123.

[4] Hoverman, J. (2011) Hard Times for Oncologists? Journal of Oncology Practice, 7, 82s-84s. https://doi.org/10.1200/JOP.2011.000416

[5] Brow, M.E., Okon, T.A., George, E.T., et al. (2012) Thoughts and Recommendations on Cancer Care Site of Service. Community Oncology, 9, 382-388. https://doi.org/10.1016/j.cmonc.2012.11.004

[6] Community Oncology Alliance. The Changing Landscape of Oncology Care. https://communityoncology.org/2020-community-oncology-alliance-practice-impa ct-report/

[7] Neubauer, M., Hoverman, J., Kolodziej, M., et al. (2010) Cost Effectiveness of Evidence-Based Treatment Guidelines for the Treatment of Non-Small-Cell Lung Cancer in the Community Setting. Journal of Oncology Practice, 6, 12-18. https://doi.org/10.1200/JOP.091058

[8] Hoverman, R., Cartwright, T., Patt, D., et al. (2011) Pathways, Outcomes, and Costs in Colon Cancer: Retrospective Evaluations in Two Distinct Databases. Journal of Oncology Practice, 7, 525-595.

[9] Kolodziej, M., Hoverman, J., Garey, J., et al. (2011) Benchmarks for Value in Cancer Care: An Analysis of a Large Commercial Population. Journal of Oncology Practice, 7, 301-306. https://doi.org/10.1200/JOP.2011.000394

[10] Hogan, C., Lunney, J., Gabel, J. and Lynn, J. (2001) Medicare Beneficiaries' Costs of Care in the Last Year of Life. Health Affairs, 20, 188-195.

[11] Emanuel, E., Ash, A., Yu, W., et al. (2002) Managed Care, Hospice Use, Site of Death, and Medical Expenditures in the Last Year of Life. Archives of Internal Medicine, 162, 1722-1728. https://doi.org/10.1001/archinte.162.15.1722

[12] Wright, A., Zhang, B., Ray, A., et al. (2008) Associations between End-of-Life Discussions, Patient Mental Health, Medical Care near Death, and Caregiver Bereavement Adjustment. JAMA, 300, 1665-1673. https://doi.org/10.1001/jama.300.14.1665

[13] Lundquist, G., Rasmussen, B. and Axelsson, B. (2011) Information of Imminent Death or Not: Does It Make a Difference? Journal of Clinical Oncology, 29, 3927 3931.

[14] Detering, K., Hancock, A., Reade, M. and Silvester, W. (2010) The Impact of Advance Cancer Planning on End of Life Care in Elderly Patients: Randomized Controlled Trial. BMJ, 340, c1345.

[15] Temel, J., Greer, J., Muzikansky, A., et al. (2010) Early Palliative Care for Patients with Metastatic Non-Small-Cell Lung Cancer. The New England Journal of Medicine, 363, 733-742. https://doi.org/10.1056/NEJMoa1000678

[16] Smith, T., Temin, S., Alesi, E., et al. (2011) American Society of Clinical Provisional Clinical Opinion: The Integration of Palliative Care into Standard Oncology Care. Journal of Clinical Oncology, 38, 5161.

[17] Hoverman, J.R., Klein, I., Harrison, D.W., et al. (2014) Opening the Black Box: The Impact of an Oncology Management Program Consisting of Level 1 Pathways and an Outbound Nurse Call System. Journal of Clinical Oncology, 10, 63-67. https://doi.org/10.1200/JOP.2013.001210

[18] Hoverman, J.R., Neubauer, M.A., Jameson, M., et al. (2018) Three-Year Results of a Medicare Advantage Cancer Management Program. Journal of Oncology Practice, 14, e229-e237.

[19] Winn, R.J. and McClure, J. (2003) The NCCN Practice Guidelines in Oncology: A Primer for Users. Journal of the National Comprehensive Cancer Network, 1, 6-13. 
https://doi.org/10.6004/jnccn.2003.0003

[20] Waters, T.M., Kaplan, C.M., Graetz, et al. (2019) Patient-Centered Medical Homes n Community Oncology Practices: Changes in Spending and Care Quality Associate with the COME HOME. Journal of Oncology Practice, 15, e56-e64.

https://doi.org/10.1200/JOP.18.00479

[21] Newcomer, L.N. (2012) Changing Physician Incentives for Cancer Care to Reward Better Patient Outcomes Instead of Use of More Costly Drugs. Health Affairs (Millwood), 31, 780-785. https://doi.org/10.1377/hlthaff.2012.0002

[22] Ellis, P.G., O’Neil, Earle, M.F., et al. (2017) Clinical Pathways: Management of Quality and Cost in the Metastatic Colorectal Cancer Setting. Journal of Oncology Practice, 13, e346-e352. https://doi.org/10.1200/JOP.2016.019232

[23] Hertler, A., Chau, S., Khetarpal, R., et al. (2020) Utilization of Clinical Pathways Can Reduce Drug Spend within the Oncology Care Model. JCO Oncology Practice, 16, e456-e463.

[24] Wilfong, L., Ginsburg, A., Hoverman, J.R., et al. (2020) Treatment Pathways for First-Line Metastatic Non-Small Cell Lung Cancer: Cost and Survival. Journal of Clinical Pathways, 6, 52-57.

[25] Dorn, S. (2020) The COVID-19 Pandemic and Resulting Economic Crash Have Caused the Greatest Health Insurance Losses in American History.

https://familiesusa.org/resources/the-covid-19-pandemic-and-resulting-economic-c rash-have-caused-the-greatest-health-insurance-losses-in-american-history/

[26] Dreyer, T., Wilfong, L.S., Patel, K., et al. (2021) Oncology Care Model: A Herculean Effort with Fixable Fatal Flaws. JCO Oncology Practice, 16, e1433-e1440.

[27] Carlson, R.W., Scavone, J.L., Koh, W., et al. (2016) NCCN Framework for Resource Stratification: A Framework for Providing and Improving Global Quality Oncology Care. Journal of the National Comprehensive Cancer Network, 14, 961-969. https://doi.org/10.6004/jnccn.2016.0103 